PAPER

Electrical conduction and dielectric relaxation properties of AIN thin films grown by hollowcathode plasma-assisted atomic layer deposition

To cite this article: Halit Altuntas et al 2016 Semicond. Sci. Technol. 31075003

View the article online for updates and enhancements.
Related content

- Atomic laver deposition: an enabling
$\frac{\text { technology for the growth of functional }}{\text { nanoscale semiconductors }}$
Necmi Biyikli and Ali Haider
- Conduction mechanism of leakage current
$\frac{\text { due to the traps in ZrO2 thin film }}{\text { Yohan Seo, Sangyouk Lee, Ilsin An et al. }}$
- Binary group III-nitride based
$\frac{\text { heterostructures: band offsets and }}{\text { transport properties }}$
Basanta Roul, Mahesh Kumar, Mohana K
Rajpalke et al.

Recent citations

Atomic laver deposition: an enabling technology for the growth of functional nanoscale semiconductors Necmi Biyikli and Ali Haider 


\title{
Electrical conduction and dielectric relaxation properties of AIN thin films grown by hollow-cathode plasma-assisted atomic layer deposition
}

\author{
Halit Altuntas $^{1}$, Turkan Bayrak ${ }^{2,3}$, Seda Kizir $^{2,3}$, Ali Haider ${ }^{2,3}$ and \\ Necmi Biyikli ${ }^{2,3}$ \\ ${ }^{1}$ Faculty of Science, Department of Physics, Cankiri Karatekin University, Cankiri 18100, Turkey \\ ${ }^{2}$ National Nanotechnology Research Center (UNAM), Bilkent University, Bilkent, Ankara 06800 Turkey \\ ${ }^{3}$ Institute of Materials Science and Nanotechnology, Bilkent University, Bilkent, Ankara 06800, Turkey \\ E-mail: altunhalit@gmail.com and biyikli@unam.bilkent.edu.tr
}

Received 10 March 2016, revised 27 April 2016

Accepted for publication 6 May 2016

Published 6 June 2016

\begin{abstract}
In this study, aluminum nitride (AIN) thin films were deposited at $200{ }^{\circ} \mathrm{C}$, on $p$-type silicon substrates utilizing a capacitively coupled hollow-cathode plasma source integrated atomic layer deposition (ALD) reactor. The structural properties of AlN were characterized by grazing incidence x-ray diffraction, by which we confirmed the hexagonal wurtzite single-phase crystalline structure. The films exhibited an optical band edge around $\sim 5.7 \mathrm{eV}$. The refractive index and extinction coefficient of the AlN films were measured via a spectroscopic ellipsometer. In addition, to investigate the electrical conduction mechanisms and dielectric properties, $\mathrm{Al} /$ $\mathrm{AlN} / p$-Si metal-insulator-semiconductor capacitor structures were fabricated, and current density-voltage and frequency dependent $(7 \mathrm{kHz}-5 \mathrm{MHz})$ dielectric constant measurements (within the strong accumulation region) were performed. A peak of dielectric loss was observed at a frequency of $3 \mathrm{MHz}$ and the Cole-Davidson empirical formula was used to determine the relaxation time. It was concluded that the native point defects such as nitrogen vacancies and $D X$ centers formed with the involvement of $\mathrm{Si}$ atoms into the AlN layers might have influenced the electrical conduction and dielectric relaxation properties of the plasma-assisted ALD grown AlN films.
\end{abstract}

Keywords: aluminum nitride, atomic layer deposition (ALD), plasma-assisted ALD, Hollowcathode plasma, current transport, dielectric

(Some figures may appear in colour only in the online journal)

\section{Introduction}

Aluminum nitride (AIN) is a member of the group III-nitride wide band gap semiconductor family, possessing the largest direct band gap with ceramic/dielectric-like properties. Growth of high quality AlN thin films on different substrates is still a subject of significant research due to their peculiar properties such as hardness, good thermal and chemical stability, high electrically resistivity, high thermal conductivity, high dielectric constant, wide band gap $(6.2 \mathrm{eV})$, low toxicity, high ultrasonic velocity, and good piezoelectric coefficient [1-10]. These good properties make AlN thin films a promising material for versatile applications in electronic, optoelectronic, piezoelectric, and acoustic devices. Featuring a band gap larger than several important conventional and high$k$ dielectrics including $\mathrm{Si}_{3} \mathrm{~N}_{4}, \mathrm{HfO}_{2}$, and $\mathrm{ZrO}_{2}$, the potential of AlN as an alternative gate dielectric material is critical. Throughout the literature, AlN thin films have been grown using various methods such as magnetron sputtering [11, 12], molecular beam epitaxy (MBE) [13, 14], metalorganic 
chemical vapor deposition (MOCVD) [15], ion-beamenhanced deposition [16, 17], plasma-enhanced chemical vapor deposition [18], and thermal or plasma-assisted atomic layer deposition (PA-ALD) [19-27]. Although epitaxy enables the deposition of high quality AIN thin films, these techniques (MBE, MOCVD) necessitate substrate temperatures typically are above $1000{ }^{\circ} \mathrm{C}$. Such a high temperature level is not compatible for post-processing on $\mathrm{Si}$ substrates used in CMOS technology. Therefore, low-temperature growth methods that might provide decent film quality need to be developed.

In recent years, atomic layer deposition (ALD), which is a special type of chemical vapor deposition (CVD) technique, has been used to deposit thin films at reduced substrate temperatures [28, 29]. Unlike CVD, in this technique, gaseous reactants (precursors) are injected into the ALD reactor as sequential pulses one at a time and separated by sufficient purging periods to eliminate gas-phase reactions. During the process, once a molecular layer of a gaseous reactant is chemisorbed on the surface of the substrate, the gas-solid reaction stops since the precursor molecules of the same kind do not react with each other. This special growth mechanism is named as 'self-limiting', which is a key property of the ALD process and enables the deposition of highly conformal and uniform thin films along with sub-monolayer control of film thickness. Moreover, plasma sources can be used to create highly reactive radicals that contribute to chemical reactions occurring at the surface, which helps to further lower the growth temperatures compared to conventional thermal ALD reactions. In our previous studies, we reported on the electrical characterization of AIN thin films deposited by an inductively coupled plasma (ICP) source assembled PA-ALD system [20-22, 30]. However, using the quartzbased ICP source led to relatively high oxygen impurities in III-nitride thin films and therefore the ICP source was replaced with a stainless-steel capacitively-coupled hollowcathode plasma (HCP) source. With this plasma source modification, we were able to achieve low-temperature selflimiting growth of crystalline $\mathrm{AlN}, \mathrm{GaN}$, and $\mathrm{Al}_{x} \mathrm{Ga}_{1-x} \mathrm{~N}$ thin films with orders of magnitude reduced oxygen impurity levels [31]. Recipe optimization, growth processes, and structural material properties were reported in that study, however the important electrical properties of interest were not addressed. In this study, our main goal is to investigate the electrical conduction and dielectric properties of AlN thin films grown at low temperatures by using hollow-cathode plasma-assisted atomic layer deposition (HCPA-ALD). In addition to these properties, the results obtained within this study make it possible to compare the influence of two different plasma sources on the properties of the PA-ALD grown AlN thin films.

\section{Experimental method}

Solvent-cleaned $\mathrm{Si}(100)$ substrates were subjected to a piranha etch $\left(\mathrm{H}_{2} \mathrm{SO}_{4}: \mathrm{H}_{2} \mathrm{O}_{2}=4: 1\right)$ for 5-10 min, which was followed by the native oxide removal in dilute hydrofluoric acid solution (HF, 2 vol.\%) for 2-3 min. Back-side ohmic contacts were formed by thermal evaporation and subsequent rapid thermal annealing. First, $\sim 80 \mathrm{~nm}$ thick $\mathrm{Al}$ was deposited to the back side of each wafer using the VAKSIS thermal evaporation system (PVD Vapor 3S Thermal), while the top wafer side was protected with a layer of photoresist. After stripping the photoresist layers and cleaning the samples with acetone, methanol, isopropanol, and deionized (DI) water, and finally drying with $\mathrm{N}_{2}$, samples were annealed in an ATV-Unitherm (RTA SRO-704) rapid thermal annealing system at $450{ }^{\circ} \mathrm{C}$ for $2 \mathrm{~min}$ under $100 \mathrm{sccm} \mathrm{N}$ flow with a ramp rate of $15^{\circ} \mathrm{C} \mathrm{s}^{-1}$. $\mathrm{Si}(100)$ substrates with back ohmic contacts and solvent-cleaned bare Si (100) substrates (as reference samples for material characterization purposes) were then loaded into a customized Fiji F200-LL ALD reactor (Ultratech/CambridgeNanotech Inc.) equipped with a capacitively coupled hollow-cathode plasma source (Meaglow Inc.) immediately after they were dipped into dilute HF solution, rinsed with DI water, and dried with $\mathrm{N}_{2}$. All depositions started with a metalorganic pulse. Trimethylaluminum $\left(\mathrm{AlMe}_{3}\right)$ and $\mathrm{N}_{2} / \mathrm{H}_{2}$ plasma were used as the $\mathrm{Al}$ and $\mathrm{N}$ precursors, respectively. $\mathrm{AlMe}_{3}$ was kept at room temperature and $5 \mathrm{~N}$-grade $\mathrm{N}_{2}$ and $\mathrm{H}_{2}$ plasma gases along with the carrier gas, Ar, were further purified using MicroTorr gas purifiers. Metalorganic precursor pulses and $\mathrm{N}_{2} / \mathrm{H}_{2}$ plasma gases (50/ $50 \mathrm{sccm}$ ) were carried from separate lines by 30 and $100 \mathrm{sccm}$ Ar, respectively. The speed of the Adixen ATH 400M turbo pump was adjusted in order to keep the reactor base pressure at around $\sim 150$ mTorr during the growth experiment. A remote RF-plasma $(300 \mathrm{~W})$ was activated at each cycle only during the flow of $\mathrm{N}$-containing plasma gas. Unless stated otherwise, the system was purged for $10 \mathrm{~s}$ after each exposure. All growth experiments were carried out at a fixed substrate temperature of $200{ }^{\circ} \mathrm{C}$.

The top contacts of the metal-insulator-semiconductor (MIS) structures were formed by another thermal evaporation ( $~ 80 \mathrm{~nm}$ thick Al) process followed by lift-off. MIS capacitor structures with AlN as the insulating layer were fabricated on $25 \mathrm{~mm} \times 40 \mathrm{~mm} p$-type $\mathrm{Si}(100)$ substrates using class 100 and 1000 cleanroom facilities. Al and AlN layers were patterned simultaneously to obtain MIS devices with $105225 \mu \mathrm{m}^{2}$ active area during the development of $\mathrm{AZ}$ 5214E photoresist with AZ $400 \mathrm{~K}$ developer (MicroChemicals GmbH) (AZ $\left.400 \mathrm{~K}: \mathrm{H}_{2} \mathrm{O}=1: 4\right)$.

The capacitance-voltage $(C-V)$ and current-voltage (I$V$ ) characteristics of the fabricated MIS capacitor structures were measured in the dark using a semiconductor parameter analyzer (Keithley 4200-SCS), which was connected to a DC probe station (Cascade Microtech PM-5). $C-V$ curves were obtained in the frequency range $7 \mathrm{kHz}-5 \mathrm{MHz}$ within a strong accumulation regime. The structural properties of HCPAALD grown AlN films were characterized via grazing incidence x-ray diffraction (GIXRD). GIXRD measurements were performed in a PANalytical X'Pert PRO MRD diffractometer using $\mathrm{CuK} \alpha$ radiation. Ellipsometric spectra of the films were recorded in the wavelength ranges 200-1200 nm by using a variable angle spectroscopic ellipsometer (J A Woollam) and optical constants of the $\sim 50 \mathrm{~nm}$ 


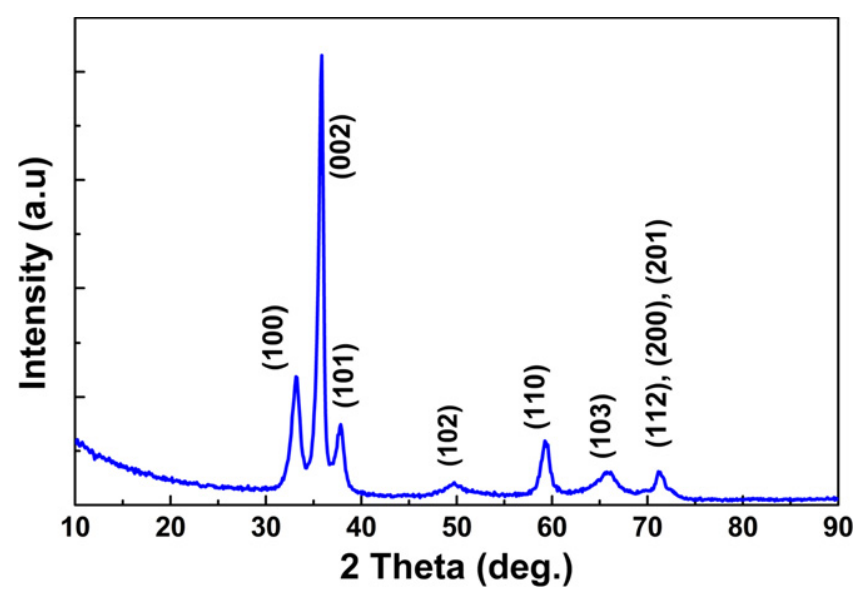

Figure 1. GIXRD pattern of a $\sim 50 \mathrm{~nm}$ thick AlN thin film deposited at $200{ }^{\circ} \mathrm{C}$ on a $\mathrm{Si}(100)$ susbtrate. The film is polycrystalline with a dominant (002) peak and hexagonal wurtzite structure.

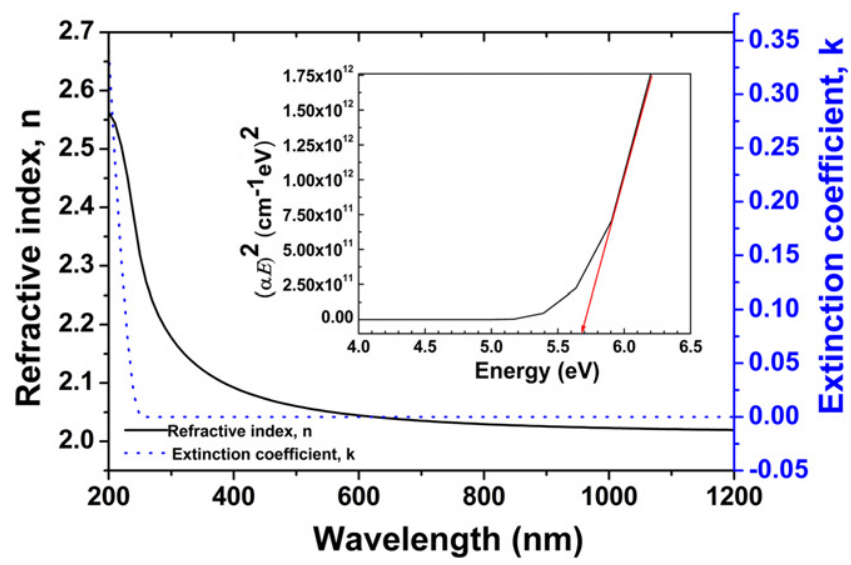

Figure 2. Optical constants (refractive index and extinction coefficient) of $\sim 50 \mathrm{~nm}$ thick AlN thin film deposited at $200{ }^{\circ} \mathrm{C}$ on aSi(100) susbtrate by HCPA-ALD. The inset figure shows the extraction of the optical band edge of AlN films.

deposited AIN thin films were modeled by the Tauc-Lorentz (TL) function, and used for the estimation of the film thicknesses. Room-temperature transmission and absorption measurements were performed with a UV-VIS spectrophotometer (Varian Cary 100) to evaluate the optical band gap of the grown AlN films.

\section{Results and discussion}

\subsection{Structural and optical properties of HCPA-ALD deposited AlN thin films}

In figure 1, GIXRD patterns of the grown AIN thin films with $\sim 50 \mathrm{~nm}$ thickness on $\mathrm{Si}(100)$ substrates are presented. All labeled Bragg diffraction peaks on the patterns correspond to reflections from the hexagonal wurtzite phase, close-to-perfectly matching with the literature (ICDD reference code: $00-$ 025-1133) database values. This result reveals that crystalline wurtzite AlN thin films can be deposited by using HCPAALD at low substrate temperatures.

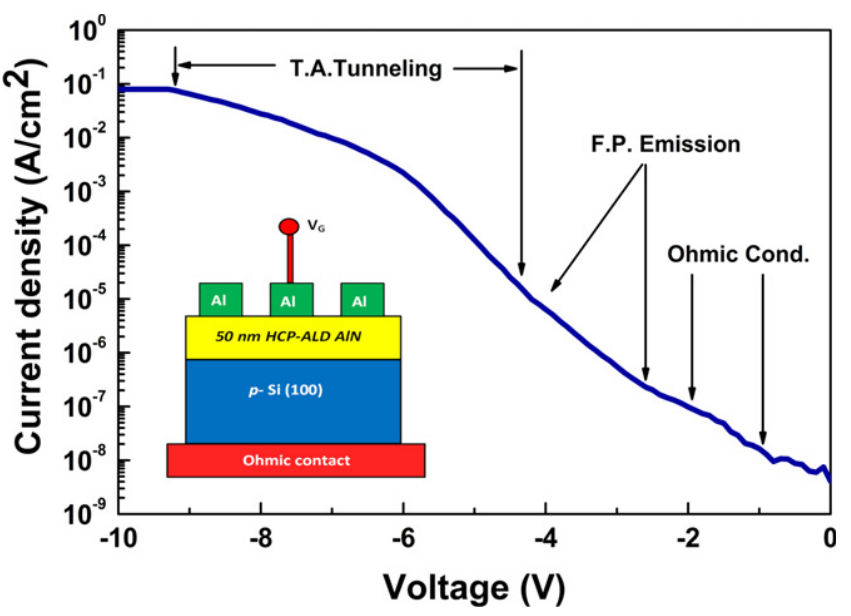

Figure 3. $J-V$ characteristics of MIS capacitors featuring HCPAALD grown AlN films as dielectric layers.

Spectral refractive index $(n)$ and extinction coefficient $(k)$ graphs of HCPA-ALD AlN thin films were obtained by spectroscopic ellipsometer measurements and shown in figure 2. As can be seen, the $n$ value is 2.56 at $200 \mathrm{~nm}$ and gradually decreases to 2.02 at $1200 \mathrm{~nm}$. As mentioned in our previous study [30], the $n$ value was measured to be 1.87 at $633 \mathrm{~nm}$ for ICP-sourced PA-ALD deposited AlN thin films. In this study, the $n$ value is 2.05 at $633 \mathrm{~nm}$, resulting in an optical dielectric constant $\left(\kappa_{\mathrm{op}}\right)$ that is equal to the square of the refractive index (i.e. $\kappa_{\mathrm{op}}=n^{2}$ ), $\kappa_{\mathrm{op}} \sim 4.2$ ) and this value is higher than ICP-sourced PA-ALD deposited AlN thin films. As is known, due to the fact that the $n$ value depends on film crystallinity, this indicates an enhancement in the crystalline quality for films deposited using the current configuration with the HCP source. On the other hand, the $k$ value decreased quite rapidly within the $200-250 \mathrm{~nm}$ wavelength range and became almost zero beyond $250 \mathrm{~nm}$, which indicates that AlN is fully transparent in the visible and nearinfrared spectrum. In addition, the optical band gap $\left(E_{g}\right)$ values of the HCPA-ALD grown AIN films were obtained by using the measured absorption spectrum and plotting of $\alpha^{2} E^{2}$ versus photon energy (inset figure of figure 2). A straight line fitted through the measured data (red arrow shown in the inset of figure 2) intersects at $\sim 5.7 \mathrm{eV}$, revealing the optical band edge of AlN thin films, which is similar to the AlN results obtained via the ICP source.

\subsection{Current conduction mechanisms in HCPA-ALD deposited AIN thin films}

To investigate the electrical transport mechanisms in HCPAALD grown AlN thin films, current density-voltage $(J-V)$ measurements were carried out on the $\mathrm{Al} / \mathrm{AlN} / p$-Si MIS capacitor structure. During the measurements, the MIS capacitor was biased in accumulation mode and the $J-V$ curve displayed is shown in figure 3 , in which the current density depends strongly on the applied electric field and several electrical conduction models were tested to explain the measured $J-V$ curves. 


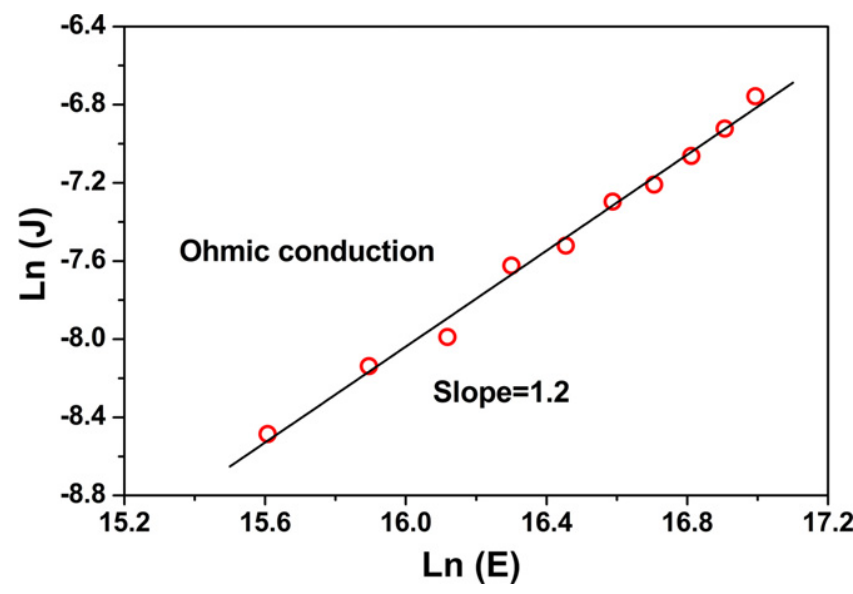

Figure 4. $\ln (J)$ versus $\ln (E)$ plot of the $\mathrm{Al} / \mathrm{AlN} / p$-Si capacitor structures.

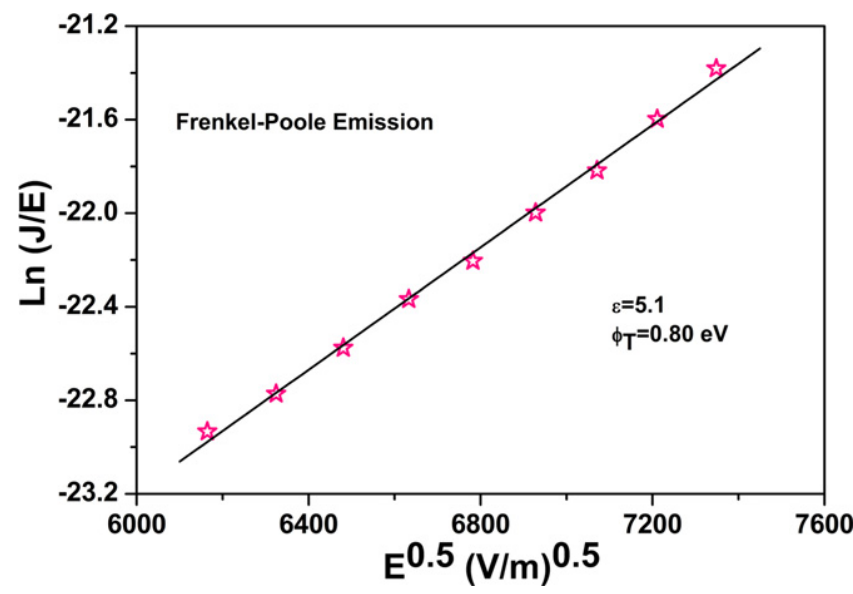

Figure 5. FP emission plot (ln $J / E$ versus $\left.E^{1 / 2}\right)$.

At low electric fields, the current density is proportional to the electric field $(E)$ and the slope of the $\ln (J)$ versus $\ln (E)$ plot (figure 4 ) is pretty close to 1 . This behavior corresponds to an ohmic type of conduction mechanism. $E$ values were calculated from $\left(V-V_{\mathrm{FB}}\right) / t_{\mathrm{AlN}}$, where $V_{\mathrm{FB}}$ is the flat-band voltage and $t_{\mathrm{AlN}}$ corresponds to the thickness of the HCPAALD grown AIN layer.

In the medium electric field regime, figure 5 shows the $\ln (J / E)$ versus $E^{1 / 2}$ plot according to the Frenkel-Poole (FP) emission theory. In this model, the current density is given as [32]

$$
J \propto E \exp \left[\frac{-q\left(\phi_{t}-\sqrt{q E / \pi \varepsilon_{d} \varepsilon_{o}}\right)}{k_{\mathrm{B}} T}\right]
$$

where $\phi_{t}$ is the trap energy level in the band gap of the dielectric material, $E$ is the electric field, $\varepsilon_{d}$ is the dynamic dielectric constant of the dielectric, $q$ is the electronic charge, $T$ is the absolute temperature, $\varepsilon_{o}$ is the vacuum dielectric constant, and $k_{\mathrm{B}}$ is the Boltzmann constant. Using equation (1), the slope in figure 5 gives an estimate of the dielectric constant of the AlN thin film. As can be seen from figure 5 , the plot is very well fitted $\left(R^{2}=0.997\right)$ with the FP mechanism, and, using equation (1), $\phi_{t}$ and $\varepsilon_{d}$ were calculated to be $0.80 \mathrm{eV}$ and 5.1,

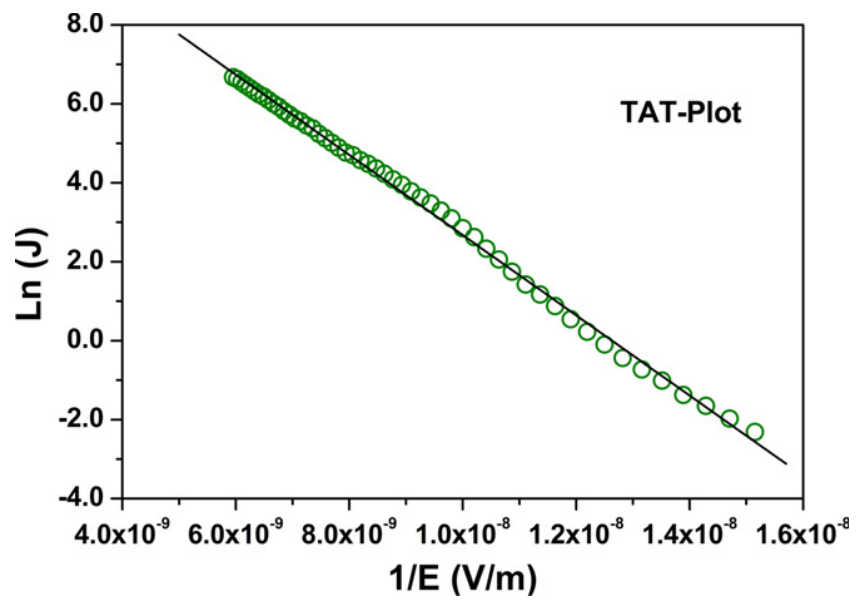

Figure 6. $\ln J$ versus $1 / E$ plot (i.e. TAT plot).

respectively. We point out that having a straight line on the $\ln$ $(J / E)$ verus $E^{1 / 2}$ plot does not guarantee FP based conduction. Therefore, it is important to ensure that the obtained dynamic dielectric constant from the slope of the $\ln J / E$ versus $E^{1 / 2}$ plot is comparable with static and optical dielectric constants.

As noted in a later section of the article, the static dielectric constant $\varepsilon_{s}$ of the HCPA-ALD grown AlN films was calculated as $\sim 6.4$ from the $C-V$ measurements at a frequency of $1 \mathrm{MHz}$. A $\sim 5.1$ dynamic dielectric constant that was obtained from equation (1) is between the optical dielectric constant (4.2) and static dielectric constant (6.4) and that means $\varepsilon_{d}$ is quite comparable with $\kappa_{\mathrm{op}}$ and $\varepsilon_{s}$. Thus, we can safely say that the current conduction mechanism in our $\mathrm{Al} /$ $\mathrm{AIN} / p$-Si MIS capacitor at medium electric fields is due to FP emission. On the other hand, some studies have reported trap states in AlN grown on $\mathrm{Si}$ substrates as $0.47-0.62 \mathrm{eV}$ and $0.73-0.80 \mathrm{eV}[33,34]$. They identified those trap states as the levels of nitrogen vacancies. In our study, the obtained trap energy level is $0.80 \mathrm{eV}$ and this value is in agreement with the reported range of $0.73-0.80 \mathrm{eV}$. Therefore, the obtained energy level of trap states can be attributed to the nitrogen vacancies in the deposited AlN films.

Finally, in the higher electric field regime, $\ln J$ versus 1/ $E$ shows a linear relationship, which possibly indicates that the conduction mechanism at those field ranges is mainly due to trap-assisted tunneling (TAT).

The current density due to the TAT mechanism is given by [32]

$$
J_{\mathrm{TAT}} \propto \exp \left\{\frac{-8 \pi \sqrt{2 q m_{\mathrm{AIN}}}}{3 h E} \phi_{t}^{3 / 2}\right\}
$$

where $\phi_{t}$ is the energy level of the electronic defects in the band gap of the dielectric material and can be obtained from a plot of $\ln J$ versus $1 / E$. Thus, $\phi_{t}$ was obtained from figure 6 as $0.38 \mathrm{eV}$.

The obtained trap energy level is obviously different from the levels of nitrogen vacancies. Ligatchev et al [33] reported energy values of $0.35-0.42 \mathrm{eV}$ in $\mathrm{AlN}$ films deposited on Si substrates by using deep-level-transient-spectroscopy measurements. Also, Goennenwein et al [35] reported 


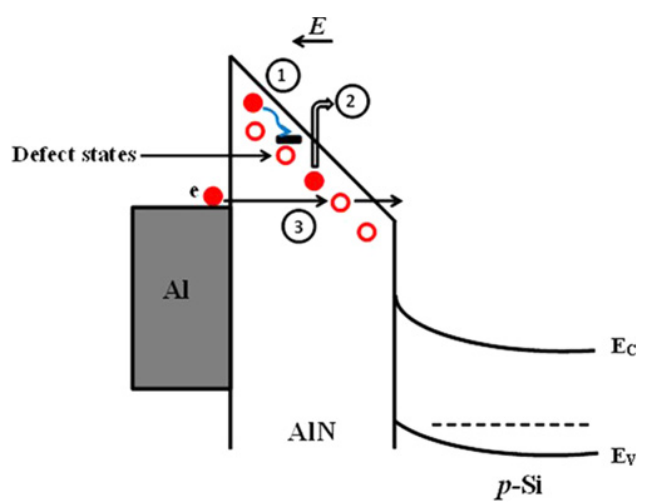

Figure 7. Band diagram of the $\mathrm{Al} / \mathrm{AlN} / p$-Si MIS structure and conduction mechanisms: (1) ohmic conduction, (2) FP emission, (3) TAT mechanism.

$0.39-0.45 \mathrm{eV}$ in Si-doped AlN films. They expressed that those defect states could be due to the so-called $D X$ centers, which are formed in AlN in the presence of $\mathrm{Si}$ atoms. The obtained trap energy level in our study is $0.38 \mathrm{eV}$, which is very close to these reported values. Therefore, we attribute these trap states to $D X$ centers. Most probably, these centers are caused by the $\mathrm{Si}$ substrate and formed near the AlN/Si interface [33]. As mentioned in our previous study [20], FP emission, TAT, and Fowler-Nordheim (FN) tunneling were found to be the electrical conduction mechanisms in the $47 \mathrm{~nm}$ thick AlN film deposited by ICP-sourced PA-ALD. Nitrogen vacancies play a role in terms of electrical conduction process in both films. But unlike ICP-sourced PAALD deposited AlN films, the trap states with small energy lavels related with $\mathrm{Si}$ atoms were obtained in the HCP-ALD deposited AlN films. Figure 7 summarizes the cumulative conduction mechanisms in HCPA-ALD deposited AlN thin films extracted from the fabricated MIS capacitors and electrical measurements.

\subsection{Dielectric relaxation characteristics of HCPA-ALD deposited AIN thin films}

We obtained capacitance-voltage $(C-V)$ curves for a range of frequencies between $7 \mathrm{kHz}$ and $5 \mathrm{MHz}$ in a strong accumulation regime and calculated the critical dielectric parameters such as relative dielectric constant $\left(\varepsilon_{r}\right)$, dielectric loss $\left(\varepsilon^{\prime \prime}\right)$, and dielectric loss tangent $(\tan \delta)$, on the basis of the formulas below [36, 37],

$$
\begin{gathered}
C_{\mathrm{AIN}}=\frac{\varepsilon_{0} \varepsilon_{r} S}{d} \\
\varepsilon^{\prime \prime}=\frac{G_{m}}{C_{0} \omega} \\
\tan \delta=\frac{\varepsilon^{\prime \prime}}{\varepsilon_{r}}
\end{gathered}
$$

where $C_{\mathrm{AlN}}$ is the capacitance of the AlN films in strong accumulation, $S$ the area of the capacitor $\left(105225 \mu \mathrm{m}^{2}\right), d$ the thickness of the AlN layer $(50 \mathrm{~nm}), G_{m}$ the measured conductance, $\omega$ the angular frequency $(=2 \pi f)$, and $C_{0}$ the capacitance of an empty capacitor $\left(=\varepsilon_{0}\left(\frac{S}{d}\right)\right)$.

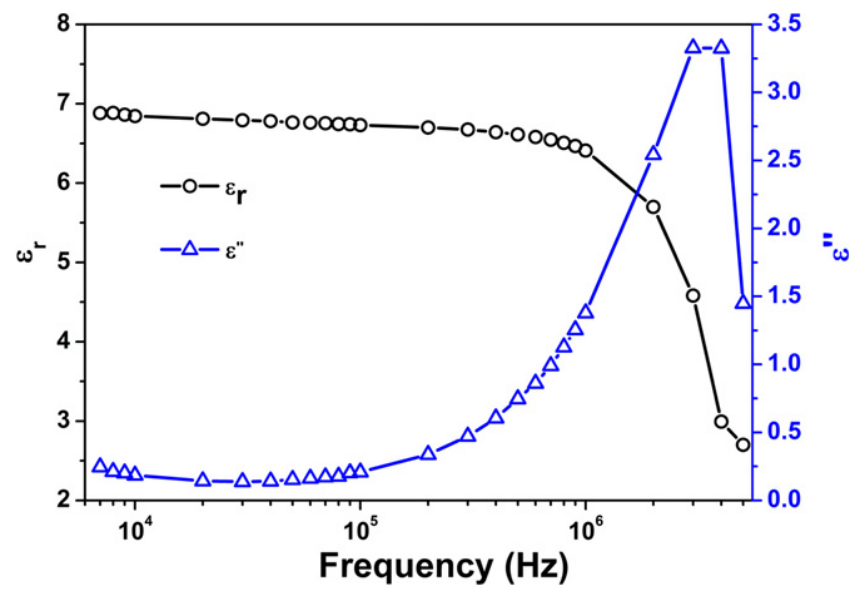

Figure 8. Room-temperature frequency dependence $(7 \mathrm{kHz}-5 \mathrm{MHz})$ of $\varepsilon_{r}$ and $\varepsilon^{\prime \prime}$ for HCPA-ALD grown AlN films.

The relative dielectric constant and dielectric loss $\left(\varepsilon_{\mathrm{r}}\right.$ and $\left.\varepsilon^{\prime \prime}\right)$ are very important physical parameters for electronic materials due to their influence on crucial electrical, optical and several other materials properties. As can be seen from figure 8 , the relative dielectric constant of AlN films was found to change between 6.88 and 2.7, which is similar to reported literature values for AIN films [38-40]. $\varepsilon_{r}$ and $\varepsilon^{\prime \prime}$ had a dependence on the measurement frequency, $f$ and $\varepsilon_{r}$ showed a decreasing trend for higher frequencies, whereas $\varepsilon^{\prime \prime}$ showed the very opposite behavior. Moreover, the dispersion curve of dielectric loss exhibited a peak around $\sim 3 \mathrm{MHz}$, which was evidence of dielectric relaxation due to orientation-dependent polarization. In this situation, the relaxation time $(\tau)$, which is a measure of the mobility of the dipoles that exist in the materials, should be determined. The relaxation frequency $f_{C}$ is inversely related to relaxation time and is defined as $\tau=\frac{1}{\omega_{C}}=\frac{1}{2 \pi f_{C}}$.

To explain the dielectric behavior due to polarization, some models have been used including the Debye model [41], the Cole-Cole model [42], and the Cole-Davidson model [43]. In this study, the best fitting performance was obtained using the Cole-Davidson model and according to this model, the relative dielectric constant $\varepsilon_{r}$ and frequency $(f)$ are expressed as

$$
\varepsilon_{r}=\varepsilon_{\infty}+\left(\varepsilon_{s}-\varepsilon_{\infty}\right)(\cos \varphi)^{\beta} \cos (\beta \varphi)
$$

and

$$
\varphi=\tan ^{-1}(\omega \tau)
$$

where $\varepsilon_{S}$ and $\varepsilon_{\infty}$ are the dielectric constants at low and high frequency limits, respectively. $\beta$ is a distribution parameter of the relaxation time $(0 \leqslant \beta \leqslant 1)$ and for $\beta=1$, equation (6) will turn into the Debye model. The smaller value of $\beta$ means a larger distribution of relaxation times. By using equations (6) and (7), the measured relative dielectric constant versus frequency data were fitted very well, as shown in figure 9. The fitted values of $\varepsilon_{S}, \varepsilon_{\infty}, \tau$, and $\beta$ were found as $6.8,1.1$, $5.2 \times 10^{-8} \mathrm{~s}$, and 0.95 , respectively. From the relaxation time, the relaxation frequency was calculated as $3.06 \mathrm{MHz}$. This value is in perfect agreement with the experimental 


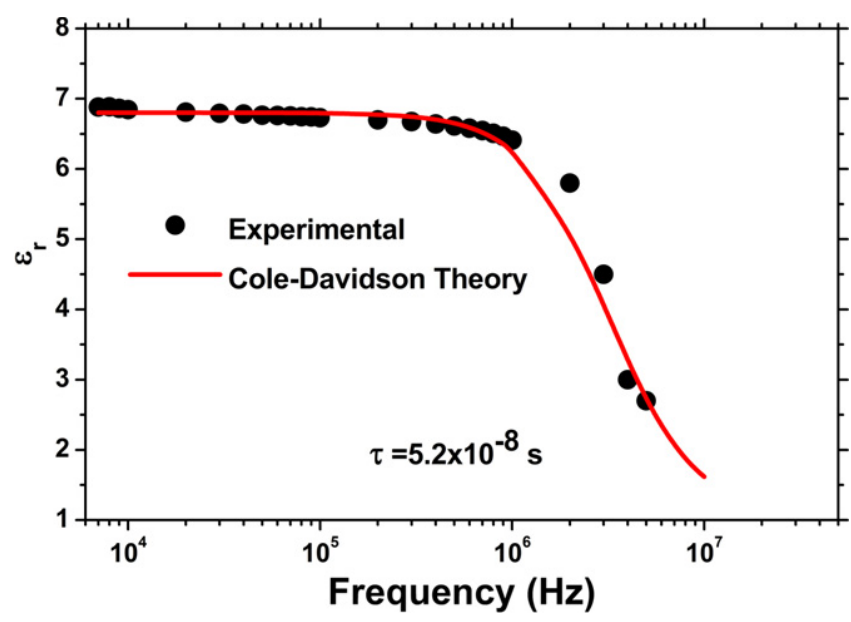

Figure 9. Fitted results of the measured relative dielectric constant $\varepsilon_{r}$ as a function of frequency with the Cole-Davidson model.

relaxation frequency $(\sim 3 \mathrm{MHz})$, which is the peak frequency of the dielectric loss. As can be seen in figure 8, the relative dielectric constant declined rapidly above the relaxation frequency $(3 \mathrm{MHz})$. This can be explained as follows. Below the relaxation frequency, the alternating electric field is slow enough and dipoles are able to keep pace with the electric field variations. However, above the relaxation frequency, dipoles are no longer able to keep pace with the applied electric field and the relative dielectric constant reduces rapidly. The obtained $\beta$ value indicates that the polarization in the HCPA-ALD deposited AIN films has not only a concrete relaxation time but also has a rather narrow as well. In Debye theory, dipoles are assumed to be independent of each other and their response to an alternating field features only one relaxation time. Nevertheless, in real materials, dipoles are more likely to be interactive (not independent), which results in a relaxation time dispersion [38].

Piezoelectric effects and point defects can cause the observed dielectric dispersion in AlN films. However, according to Nakayama et al [44], the peak of dielectric loss caused by piezoelectric effects is positioned in microwave frequencies. On the other hand, native point defects in AlN occur due to some unintentional dopants and nitrogen vacancies. These point defects may form dipoles with each other, which might influence the dielectric behavior [37, 38, 45]. Xu et al [40], observed a peak of dielectric loss at $1.5 \mathrm{MHz}$ for AlN films and the dielectric behavior of the films was attributed to dipoles related to nitrogen vacancies. Butcher and Tansley [46] reported the dielectric dispersion of AlN films grown on Si where they observed a peak of dielectric loss at $1 \mathrm{MHz}$ at $100 \mathrm{~K}$ which was attributed to diffused Si atoms in AlN. In our study, as mentioned previously, current transport mechanisms were associated with the native defects which were nitrogen vacancies and $D X$ centers formed with the involvement of substrate ( $\mathrm{Si}$ ) atoms in the AlN layers. So, we conclude that the dielectric relaxation behavior in the HCPA-ALD grown AIN thin films originates from the dipoles related to nitrogen vacancies and $D X$ centers formed with $\mathrm{Si}$ atoms.

\section{Conclusion}

Polycrystalline wurtzite AlN thin films with highly orientated (002) preferential planes were deposited on $p$-Si substrates at $200{ }^{\circ} \mathrm{C}$ by HCPA-ALD. The refractive index and optical band gap of the deposited films were found to be 2.05 (at $632 \mathrm{~nm}$ ) and $\sim 5.7 \mathrm{eV}$, respectively. To evaluate the current transport mechanisms, $\mathrm{Al} / \mathrm{AlN} / p$-Si MIS capacitor devices were fabricated and $I-V$ measurements were carried out. Ohmic conduction, FP emission, and trap-assisted tunneling were determined to be the main electrical transport mechanisms. The obtained trap levels in the films were attributed to nitrogen vacancies and Si-related $D X$ centers. As mentioned in our previous study, FP emission, TAT, and FN tunneling were found to be the basic electrical conduction mechanisms in the $47 \mathrm{~nm}$ thick AlN film deposited by ICP-sourced PAALD. Nitrogen vacancies play a role in terms of electrical conduction process in both films. However, the trap states with small energy levels related to $\mathrm{Si}$ atoms were obtained in the HCP-ALD deposited AIN films. On the other hand, we obtained the dielectric behavior of the HCPA-ALD grown AlN films by $C-V$ measurements up to $5 \mathrm{MHz}$. The relative dielectric constant of the AIN films was relaxed from 6.89 to 2.7 with increasing frequency and dielectric loss showed a peak at a frequency of $\sim 3 \mathrm{MHz}$. Using the Cole-Davidson equation, which is based on the Debye model, experimental relative dielectric constant $\varepsilon_{\mathrm{r}}$ versus frequency $(f)$ curves were fitted very well and the extracted relaxation frequency was in perfect agreement with the experimental value. This agreement indicates that the dielectric relaxation time has a rather small dispersion $(\beta=0.95)$. According to these results and comparing with other reported studies, we conclude that the dielectric behavior of HCPA-ALD grown AlN films is caused mainly by the point defects relating to nitrogen vacancies and $D X$ centers formed with diffused $\mathrm{Si}$ atoms. Those defects probably form as dipoles and influence the dielectric relaxation in the AlN layers.

\section{Acknowledgments}

This work was performed at UNAM supported by the State Planning Organization (DPT) of Turkey through the National Nanotechnology Research Center Project. N Biyikli acknowledges a Marie Curie International Reintegration Grant (IRG) for funding the NEMSmart (PIRG05-GA-2009249196) project.

\section{References}

[1] Hsu W F, Kao H L and Lin Z P 2016 J. Cryst. Growth 43646

[2] Sun M S, Zhang J C, Huang J, Wang J F and Xu K 2016 J. Cryst. Growth 43662

[3] Oikawa H et al 2015 Thin Solid Films 574110

[4] Hassine N B, Mercier D, Renaux P, Parat G, Basrour S, Waltz P, Chappaz C, Ancey P and Blonkowski S 2009 J. Appl. Phys. 105044111 
[5] Chiu K H, Chen J H, Chen H R and Huang R S 2007 Thin Solid Films $\mathbf{5 1 5} 4819$

[6] Strite S and Morkoc H 1992 J. Vac. Sci. Technol. B 101237

[7] Salmagne S R and Monch W 1995 Surf. Sci. 331-333 937

[8] Dubois M A and Mulart P 1999 Appl. Phys. Lett. 743032

[9] Ramanan N, Lee B and Misra V 2015 Semicond. Sci. Tech. 30 125017

[10] Lee Y C, Kao T T and Shen S C 2015 Semicond. Sci. Tech. 30 045010

[11] Adam T, Kolodzey J, Swann C P, Tsao M W and Rabolt J F 2001 Appl. Surf. Sci. 175-176 428

[12] Engelmark F, Westlinder J, Iriarte G F, Katardjiev I V and Olsson J 2003 IEEE Trans. Electron Devices 501214

[13] Jose F, Ramaseshan R, Dash S, Bera S, Tyagi A K and Raj B J. Phys. D.: Appl. Phys. 43075304

[14] Nechaev D V, Aseev P A, Jmerik V N, Brunkov P N, Kuznetsova Y V, Sitnikova A A, Ratnikov V V and Ivanov S V 2013 J. Cryst. Growth. 378319

[15] Tanaka Y, Hasebe Y, Inushima T, Sandhu A and Ohoya S 2000 J. Cryst. Growth. 209410

[16] An Z, Men C, Xu Z, Chu P K and Lin C 2005 Surf. Coat. Tech. 196130

[17] Men C and Lin C 2006 Mater. Sci. Eng. B 133124

[18] Sánchez G, Wu A, Tristant P, Tixier C, Soulestin B, Desmaison J and Alles B A 2008 Thin Solid Films $\mathbf{5 1 6} 4868$

[19] Ozgit C, Donmez I, Alevli M and Biyikli N 2012 Thin Solid Films $\mathbf{5 2 0} 2750$

[20] Altuntas H, Ozgit-Akgun C, Donmez I and Biyikli N 2015 IEEE Trans. Electron Devices 623627

[21] Alevli M, Ozgit C, Donmez I and Biyikli N 2012 Phys. Status Solidi a 209266

[22] Alevli M, Ozgit C, Donmez I and Biyikli N 2012 J. Vac. Sci. Technol. A 30021506

[23] Motamedi P and Cadien K 2015 J. Crystal Growth 42145

[24] Mattila P, Bosund M, Jussila H, Aierkena A, Riikonen J, Huhtio T, Lipsanen H and Sopanen M 2014 Appl. Surf. Sci. 314570

[25] Chen K J and Huang S 2013 Semic. Sci. Tech. 28074015
[26] Kueck D, Leber P, Schmidt A, Speranza G and Kohn E 2010 Diam. Relat. Mater. 19932

[27] Bosund M, Sajavaara T, Laitinen M, Huhtio T, Putkonen M, Airaksinen V M and Lipsanen H 2011 Appl. Surf. Sci. 2577827

[28] Leskela M, Niinisto J and Ritala M 2014 Comph. Mater. Process 4101

[29] Ritala M, Leskelä M, Nykänen E, Soininen P and Niinistö L 1993 Thin Solid Films 225288

[30] Altuntas H, Ozgit-Akgun C, Donmez I and Biyikli N 2015 J. Appl. Phys. 117155101

[31] Ozgit-Akgun C, Goldenberg E, Okyay A K and Biyikli N 2014 J. Mat. Chem. C 22123

[32] Sze S M 1981 Physics of Semiconductors (New York: Wiley)

[33] Ligatchev V, Rusli and Pan Z 2005 Appl. Phys. Lett. 87 242903

[34] Jenkins D W and Dow J D 1989 Phys. Rev. B 393317

[35] Goennenwein S T B, Zeisel R, Ambacher O, Brandt M S, Stutzmann M and Baldovino S 2001 Appl. Phys. Lett. 792396

[36] Daniel V V 1967 Dielectric Relaxation (London: Academic Press)

[37] Symth C P 1955 Dielectric Behavior and Structure (New York: McGraw-Hill)

[38] Bi Z X, Zheng Y D, Zhang R, Gu S L, Xiu Q, Zhou L-L, Shen B, Chen D J and Shi Y 2004 J. Mater. Sci. 15317

[39] VasanthiPillay V and Vijayalaksmi K 2012 J. Miner. Mater. Charact. Eng. 11724

[40] Xu X H, Zhang C J and Jin Z H 2001 Thin Solid Films 38862

[41] Debye P 1929 Polar Molecules (New York, NY, USA: Chemical Catalogue Company)

[42] Cole K S and Cole R H 1941 J. Chems. Phys. 9341

[43] Davidson D W and Cole R H 1950 J. Chems. Phys. 181417

[44] Nakayama A, Nambu S, Inagaki M, Miyauchi M and Itoh N 1996 J. Am. Ceram. Soc. 791453

[45] Goldsby J C 2001 J. Alloys Compd. 32167

[46] Butcher K S A and Tansley T L 2001 J. Appl. Phys. 906218 